\title{
Topological vectors as a fingerprinting system for 2D-material flake distributions
}

\author{
Joyce C. C. Santos ${ }^{1}$, Mariana C. Prado iD $^{2}$, Helane L. O. Morais $\mathbb{D}^{3}$, Samuel M. Sousa ${ }^{3}$, Elisangela Silva-Pinto ${ }^{3}$, Luiz G. Cançado $\mathbb{D}^{1,4}$ and $^{2}$
} Bernardo R. A. Neves (iD)

The production of 2D material flakes in large quantities is a rapidly evolving field and a cornerstone for their industrial applicability. Although flake production has advanced in a fast pace, its statistical characterization is somewhat slower, with few examples in the literature which may lack either modelling uniformity and/or physical equivalence to actual flake dimensions. The present work brings a methodology for 2D material flake characterization with a threefold target: (i) propose a set of morphological shape parameters that correctly map to actual and relevant flake dimensions; (ii) find a single distribution function that efficiently describes all these parameter distributions; and (iii) suggest a representation system-topological vectors-that uniquely characterizes the statistical flake morphology within a given distribution. The applicability of such methodology is illustrated via the analysis of tens of thousands flakes of graphene/graphite and talc, which were submitted to different production protocols. The richness of information unveiled by this universal methodology may help the development of necessary standardization procedures for the imminent 2D-materials industry.

npj 2D Materials and Applications (2021)5:51; https://doi.org/10.1038/s41699-021-00234-z

\section{INTRODUCTION}

The much-anticipated "2D-materials" revolution heavily relies on the availability of industrial-scale quantities of well-characterized 2D material flakes ${ }^{1,2}$. One of the most promising and scalable production methods is liquid-phase exfoliation $(\mathrm{LPE})^{3-5}$. Previous works investigating statistical distributions of 2D material flakes produced via LPE have suggested that some morphological parameters seem to be normally distributed, while others are heavily skewed ${ }^{6-8}$. Different distribution functions were employed to model distinct morphological parameters ${ }^{6-8}$, which may have weak correspondence with actual flake dimensions that are relevant for several separation and filtration processes 9 . Here, we propose an analysis methodology based on an original set of morphological parameters that effectively map to actual and relevant flake dimensions. These parameters are extracted from a single distribution function and the information is summarized by a representation system, the topological vectors, that uniquely characterizes the statistical flake morphology within a given distribution.

Flakes produced via LPE of 2D materials are far from being isotropic objects ${ }^{1-8}$. Due to the large differences between in-plane and out-of-plane separation energies (the former being much larger than the latter), flake thickness usually is much smaller than any of its lateral lengths ${ }^{7,8}$. Additionally, random shear/tear forces during exfoliation produce a broad and non-uniform distribution of flake lateral lengths. In other words, an actual flake has a rather complex polygonal geometry, which may not be realistically described by simple geometric areal shapes like rectangles or ellipses. Additionally, the use of "effective area shapes" yields lateral dimensions that do not correspond to any actual dimension of a given flake. Since the use of a large number of lateral lengths to accurately describe flake areal geometry is not practical, two characteristic lateral lengths are often used due to their relevance to several processes in different fields, from particle filtration to tumor evaluation: the maximum and minimum lateral calipers (or gauges) ${ }^{9-12}$. The maximum caliper, also known as Feret length (or diameter), is the largest distance between two parallel tangential lines in any inplane direction of a flake ${ }^{9}$. For example, during filtration, a sieve with pore diameter larger than the Feret length would never retain such flake. The minimum caliper, also known as MinFeret length or Breadth, represents the smallest distance between two tangential parallel lines in any in-plane direction of a flake'. A filter with pore diameter smaller than the MinFeret length of a flake will certainly retain it. Therefore, Feret and MinFeret lengths are key parameters in any flake separation/filtration process. In an unrelated field, the Feret/MinFeret ratio is an important parameter in the differentiation between benign and malignant tumors ${ }^{10-12}$.

In a first approximation, it might seem reasonable to use a flake's thickness, Feret and MinFeret values as its morphological characterization parameters. However, there is an important additional parameter: flake volume $\mathrm{V}$, which is strongly correlated with these three values - the larger the former, the larger the latter. A natural solution to account for this correlation is, therefore, defining three distinct dimensionless aspect ratios: $r_{h}, r_{\text {Feret, }}$ and $r_{\text {MinF, }}$, where $r_{h}=\frac{h}{V^{1 / 3}}, r_{\text {Feret }}=\frac{\text { Feret }}{V^{1 / 3}}$ and $r_{\text {MinF }}=\frac{\text { MinFeret }}{V^{1 / 3}}$. These three shape parameters can be directly mapped into the flake's actual and relevant dimensions. In other words, given the 3D image of a flake, as readily produced via atomic force microscopy (AFM), these three parameters are accurately and straightforwardly determined for that flake. Therefore, they should constitute an interesting basis set for a threefold (or three-dimensional) shape characterization system.

\section{RESULTS AND DISCUSSIONS}

AFM imaging and morphological parameters

This idea was tested for four different liquid-phase exfoliated 2D material flake products. These products comprise two distinct 2D

\footnotetext{
${ }^{1}$ MGGrafeno-CDTN/CNEN, Belo Horizonte, Minas Gerais, Brazil. ${ }^{2}$ Physics Department—Universidade Federal de Ouro Preto, Ouro Preto, Minas Gerais, Brazil. ${ }^{3}$ Instituto Federal de Minas Gerais, Campi Santa Luzia and Ouro Preto, Ouro Preto, Minas Gerais, Brazil. ${ }^{4}$ Physics Department-Universidade Federal de Minas Gerais, Belo Horizonte, Minas Gerais, Brazil. ${ }^{凶}$ email: bernardo@fisica.ufmg.br
} 

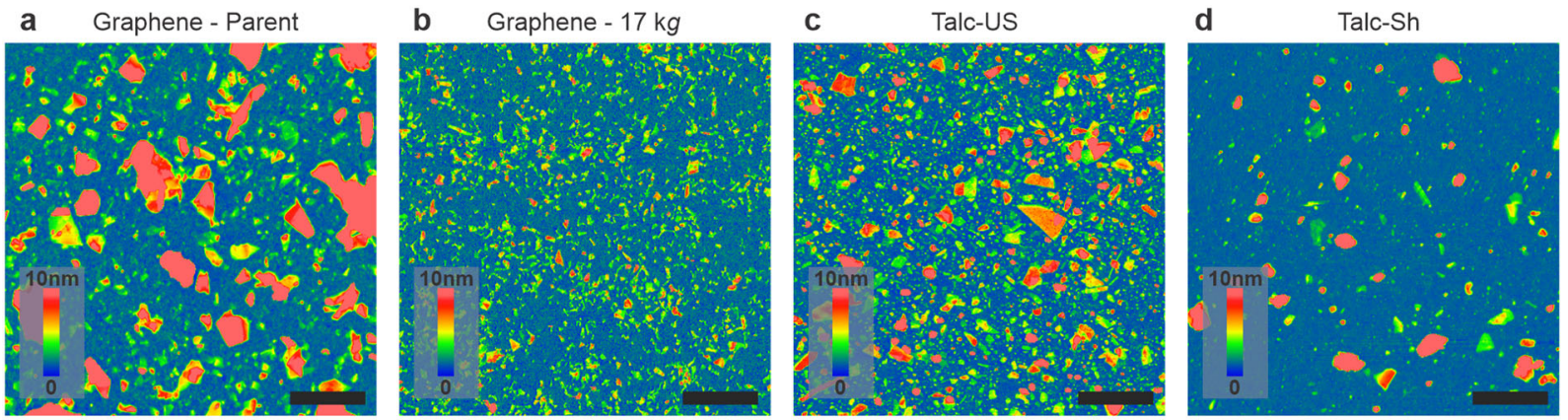

Fig. 1 Atomic force microscopy images of all four investigated systems. Panels $\mathbf{a}, \mathbf{b}$ show a representative AFM image of the graphene Parent $(\mathbf{1 7} \mathbf{~ k g})$ sample, while panels $\mathbf{c}$, $\mathbf{d}$ show a typical AFM image of the ultrasound- (shear-) produced talc sample Talc-US (Talc-Sh). The 1$\mu \mathrm{m}$-long black scale bars in all images indicate their lateral dimensions, while the colored vertical bars indicate sample height (from 0 to $10 \mathrm{~nm})$.

materials, graphene and talc, which underwent through diverse LPE processes (shear or sonication, centrifugation and specific surfactants) - see Methods section for sample preparation details. Figure 1 shows representative AFM images of all four systems investigated here. The graphene samples, named Parent and $17 \mathrm{~kg}$, were produced via the same shear/surfactant process (Fig. 1a, b, respectively). Their only difference is that the $17 \mathrm{~kg}$ sample went through an additional centrifugation step at $17 \mathrm{~kg}$ acceleration $\left(g=9.806 \mathrm{~m} / \mathrm{s}^{2}\right)$. One of the talc samples was produced via surfactant-enhanced ultrasonication (Talc-USFig. 1c) and the other was produced via a similar shear/surfactant process of the graphene samples (named Talc-Sh-Fig. 1d). The AFM images in Fig. 1 readily reveal a large variation in flake's size and shape for all systems. In order to characterize them, we took advantage of an AFM protocol that enables the statistical analysis of thousands of individual flakes ${ }^{13}$. Therefore, for each system, more than 10,000 flakes were analyzed, which required several AFM images for each case and enabled a robust and statistically representative picture of all systems. Figure $2 a, 2 b$ shows $3 D$ plots of average thickness $\boldsymbol{h}$, maximum caliper Feret and minimum caliper MinF for each flake for both graphene and talc systems, respectively. These plots and their respective projections along defined planes readily reveal some significant differences among these systems. For the graphene case (Fig. 2a), centrifugation filters out not only larger flakes (as expected), but also removes the smallest flakes, which is somewhat unexpected, but previously reported $^{8}$. For the talc case, (Fig. 2b) the values of all three parameters ( $h$, Feret and MinF) seem to be less spread for the TalcSh sample than for the Talc-US one. Even though it is possible to make several analyses based on Fig. $2 a, b^{6-8}$, there is an important morphological parameter missing in such representation: flake volume $V$ (in an AFM image, flake volume $V$ is defined as the product of flake area $A$ by its average thickness $h, V=A$.h).

As discussed above, this problem may be taken care of by the use of dimensionless aspect ratios $r_{h}, r_{F e r e t}$ and $r_{\text {MinF. }}$. Additionally, following the work of Chacham et al. ${ }^{8}$, which suggests that the natural logarithm of some quantities may be normally distributed, it seemed appropriate to investigate the probability distributions of the logarithm of these aspect ratios: $\ln \left(r_{h}\right), \ln \left(r_{\text {Feret }}\right)$, and $\ln \left(r_{\text {MinF }}\right)$. Thus, Fig. 2c, d, e shows normalized histogram distributions of these parameters for all four systems investigated here (Parentblack squares; $17 \mathrm{~kg}$-red circles; Talc-US-blue up triangles; Talc-Sh-green down triangles). It is evident that all distributions have a well-defined peak, i.e., the most-probable value for a given shape parameter. They also present different degrees of symmetry around the peak, with asymmetric tails being the most common cases. Since the definitions of all three aspect ratios $\left(r_{h}, r_{F e r e t}\right.$ and $r_{\text {MinF }}$ ) are similar, it also seemed natural and practical to employ a single model to fit all distributions, regardless their degree of asymmetry. A symmetric distribution of $n$ data points around a peak is often well described by a center parameter (e.g., mean $m$ ) and a spread parameter (e.g., standard deviation s) like, for example, a Gaussian function ${ }^{14}$. However, nonsymmetric (skewed) distributions require an additional third parameter: the skewness $\gamma$, which is defined as $\gamma=\frac{X}{s^{3}}$, where $X$ is the third moment about the mean $m\left(X=\frac{1}{n} \sum_{i=1}^{n}\left(x_{i}-m\right)^{3}\right)^{14}$. Several skewed distribution functions have been proposed to efficiently model such data and the exponentially modified Gaussian (EMG) is one of the simplest and widely used in different fields from cell biology, radiotherapy and chromatography to online game modelling ${ }^{15-20}$. It describes the sum $Z$ of independent normal $X$ and exponential $Y$ random variables, $Z=X+Y$, where $X$ is a Gaussian distribution with mean $\mu$ and variance $\sigma^{2}$ and $Y$ is an exponential distribution with decay rate $\lambda^{15-20}$. The probability density function $f$ of the EMG distribution is:

$f(x ; \mu, \sigma, \lambda)=\frac{\lambda}{2} e^{\frac{\lambda}{2}\left(2 \mu+\lambda \sigma^{2}-2 x\right)}\left[1-\operatorname{erf}\left(\frac{\mu+\lambda \sigma^{2}-x}{\sqrt{2} \sigma}\right)\right]$

where $\operatorname{erf}(x)$ is the error function ${ }^{14}$. Due to its simplicity and general character, Eq. 1 was chosen to fit all normalized probability distributions in Fig. 2 c, d, e. Fitted $R^{2}$ values $>0.99$ for all cases attest the suitability of the EMG function to accurately describe the behaviors of all three aspect ratios. Besides the normalized vertical axis, all plots in Fig. $2 c$, d, e cover the same range in the horizontal axis, which enable some straightforward comparisons among them (for this purpose, the absolute value of $\ln \left(r_{h}\right)$ was plotted in Fig. $\left.2 c\right)$. For example, it is easy to verify that the thickness-related aspect ratio $r_{\mathrm{h}}$ shows the widest distribution among the three ratios for any system. In other words, for any material or production method, the observed relative variation in flake thickness is notably larger than the variation of both characteristic lateral lengths. It is also noteworthy that the flake minimum caliper $\left(r_{\text {MinF }}\right)$ distribution is the narrowest and most symmetrical for all four systems, while the maximum caliper $\left(r_{\text {Feret }}\right)$ presents the most asymmetric distributions, with pronounced tails beyond the peak position. All these features are closely related to delamination/tear mechanisms during $\mathrm{LPE}^{6-8,21}$ and, therefore, these three dimensionless aspect ratios should form an interesting basis set for the analysis and optimization of LPE processes.

\section{The topological vectors}

Although any system can be characterized by these three distributions in Fig. 2c, d, e, it would be beneficial, from both industrial and scientific viewpoints, that a system (or sample) could be representatively depicted by a single graph (or plot) that summarizes all the relevant information. This can be accomplished with the use of a three-dimensional vectorial representation of the 
a

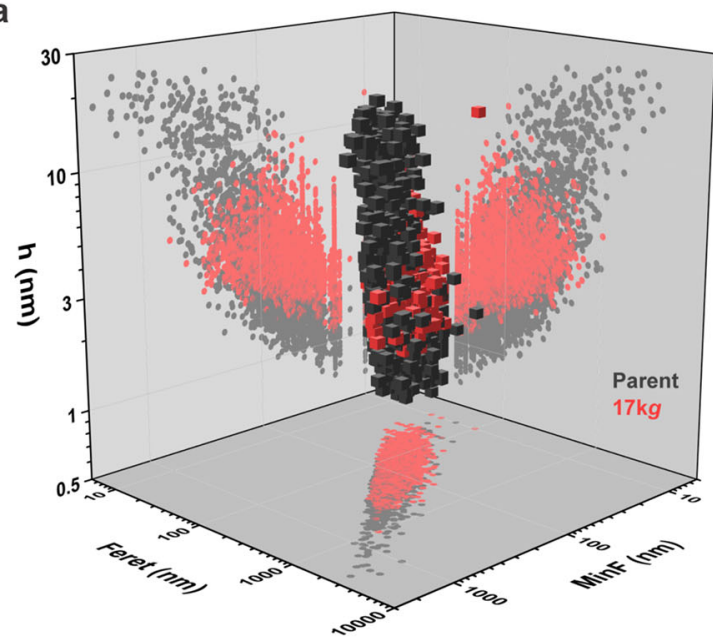

b

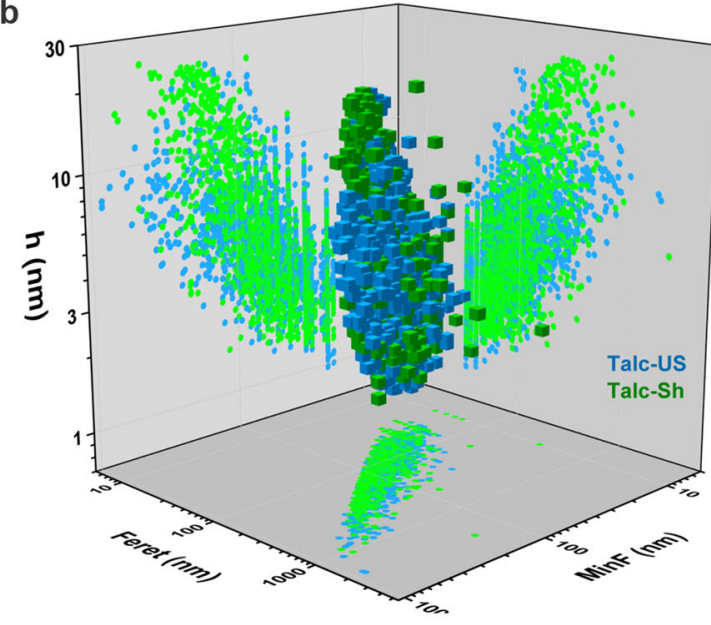

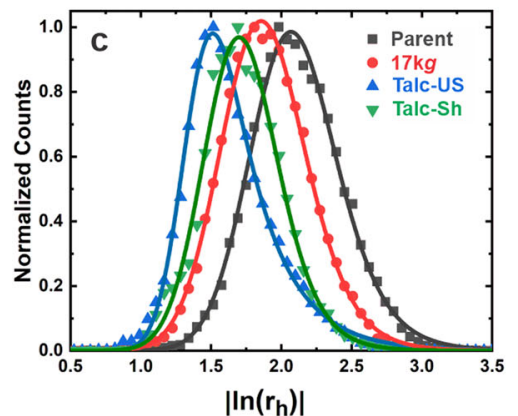
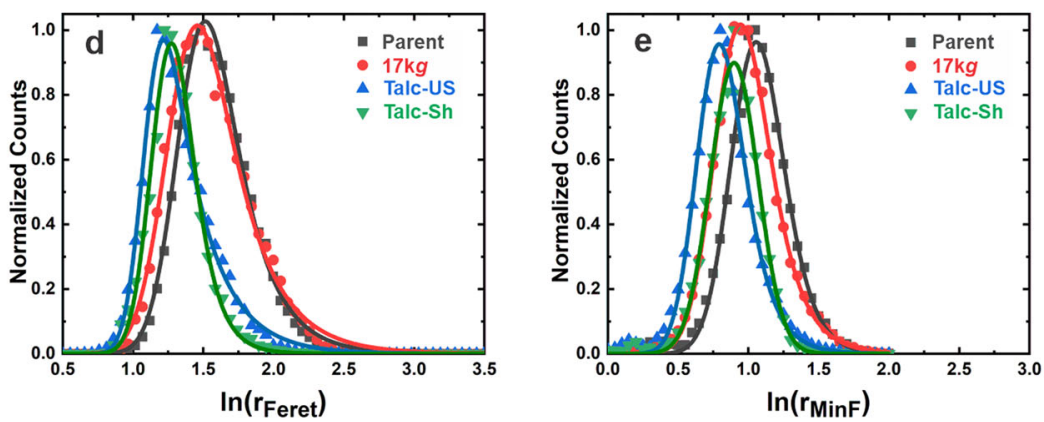

$\mathbf{f}$
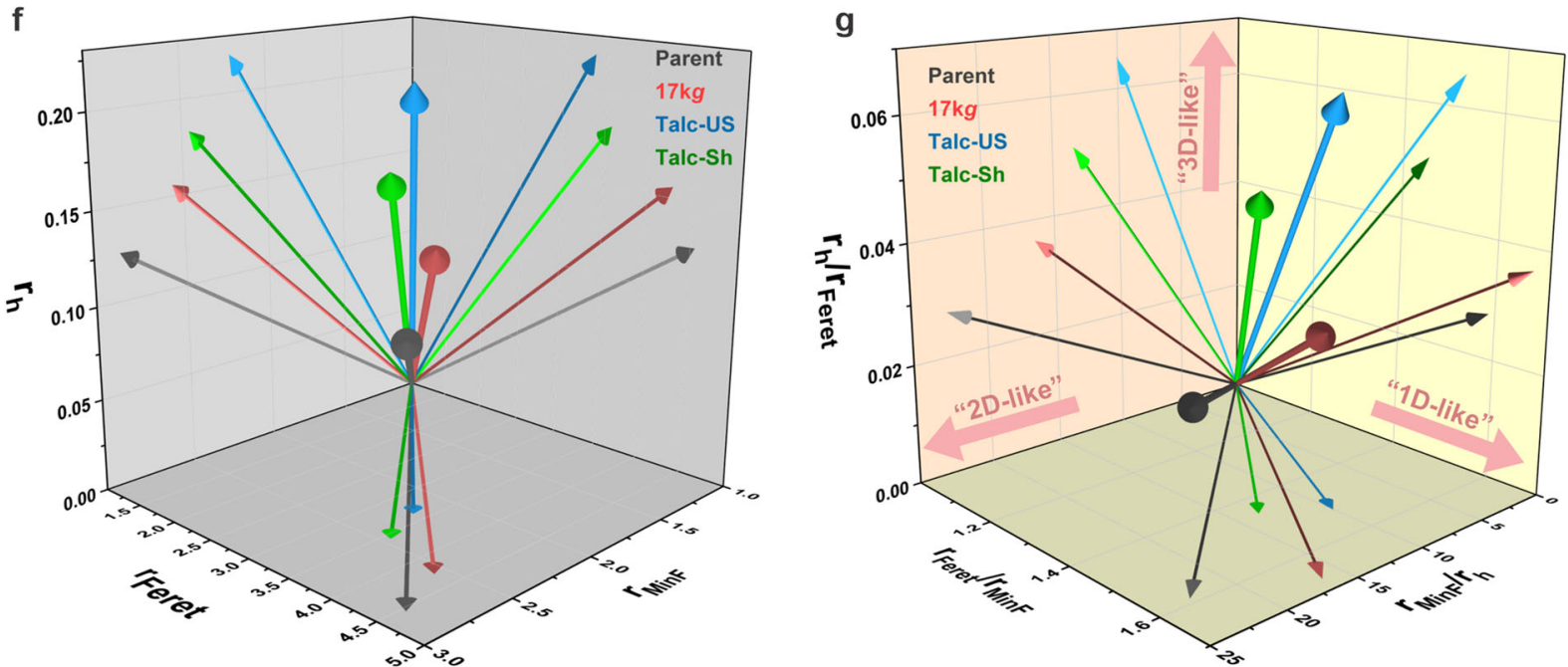

Fig. 2 Characteristic lengths, normalized probability distributions, and topological vectors of dimensionless aspect ratios $\mathbf{r}_{\mathrm{h}}, \mathbf{r}_{\mathrm{Feret}}$, and $\mathbf{r}_{\text {MinF }}$ for graphene and talc flakes. $\mathbf{a}, \mathbf{b} 3 \mathrm{D}$ plots of characteristic lengths (average height, maximum, and minimum calipers) for more than 10,000 flakes each of two graphene systems and two talc systems, respectively. The projections along each plane are also shown. c-e Normalized distributions of $\ln \left(r_{h}\right), \ln \left(r_{\text {Feret }}\right)$ and $\ln \left(r_{\text {MinF }}\right)$, respectively, for each system (Parent-black squares; $17 \mathrm{~kg}$-red circles; Talc-USblue up triangles; Talc-Sh-green down triangles). For the $r_{h}$ case, the absolute value of the logarithm was plotted to enable a direct comparison with the other two distributions. $\mathbf{f}$ Three-dimensional topological vector representation of $r_{h}$, $r_{F e r e t}$, and $r_{\text {MinF }}$ for all four systems, with projections along each plane. $\mathbf{g}$ Topological vectors and their respective plane projections of three pairwise ratio combinations of $\mathrm{r}_{\mathrm{h}}, \mathrm{r}_{\mathrm{Feret}}$, and $\mathrm{r}_{\text {MinF. }}$

distributions using the most-probable value (or the mode) as the representative value for each vector component. Such vectorial space, formed by three independent directions $\hat{r}_{\mathrm{h}}, \hat{r}_{\text {Feret, }}$ and $\hat{r}_{\text {MinF }}$, is a topological vectorial space since each vector can be transformed via topological operations into another vector. Figure $2 f$ shows, therefore, the topological vectors that represent each investigated system (Parent-black; $17 \mathrm{~kg}$-red; Talc-US—blue, and Talc-Sh-green) together with their projections along the defined planes. The differentiation efficiency of the vectorial representation is evident, as it clearly distinguishes all four systems. Such representation, including vector projections, also enables a more straightforward visual representation of each 
system's characteristic morphology. For example, the graphene Parent sample has the smallest thickness-related ratio $r_{h}$ and the largest lateral ratios $r_{\text {Feret }}$ and $r_{\text {MinF }}$, making it the most "twodimensional" system. On the other extreme, the Talc-US system portrays the largest $r_{h}$ and smallest lateral ratios, constituting, thus, the least "two-dimensional", or more 3D-like, system. It is evident that any modification on system characteristic morphology, due to, for example, an alteration in the production process, would readily change its topological vector. In other words, the topological vector and its projections constitute a fingerprinting system for the specification of a characteristic system morphology.

Additional vectorial spaces can be defined via simple mathematical operations among vector components $\hat{r}_{\mathrm{h}}, \hat{r}_{\text {Feret, }}$, and $\hat{r}_{\text {MinF. }}$ For example, topological vectors of pairwise ratios among these components can be defined, which also reveal interesting shaperelated features of these systems. Figure $2 \mathrm{~g}$ shows an example of such topological vectors for the case where the vector components are $\hat{r}_{\text {Feret }} / r_{\text {MinF, }} \hat{r}_{\text {MinF }} / r_{\mathrm{h}}$ and $\hat{r}_{\mathrm{h}} / \mathrm{r}_{\text {Feret }}$. Many analyses within each projection plane are possible, but to illustrate the potential of this representation system, only a few simple analyses on each plane will be carried out here. Initially, considering the $\hat{r}_{\text {Feret }} / r_{\text {MinF }} X$ $\hat{r}_{\mathrm{h}} / \mathrm{r}_{\text {Feret }}$ plane (light yellow shades in Fig. $2 \mathrm{~g}$ ), it is important to note that both material systems (graphene and talc) are clearly differentiated, forming two distinct sub-groups. Shape-wise, the $\mathrm{r}_{\mathrm{h}} / \mathrm{r}_{\text {Feret }}$ axis may be directly correlated with the voluminosity (or a "3D-like" shape) of a flake, while the $r_{\text {Feret }} / r_{\text {MinF }}$ axis correlates with a dominant lateral flake dimension, or a more "1D-like" or "ribbonshaped" flake-see pink arrows in Fig. 2g. Analyzing the graphene samples within such framework reveals interesting features: as the Parent graphene sample is centrifuged, creating the $17 \mathrm{~kg}$ sample, the characteristic flake shape changes. Interestingly, the centrifuged sample has a more "3D-like" or "voluminous" aspect ratio, while, at the same time, it has a larger in-plane lateral aspect ratio (or a more "ribbon-like" or "1D-like" shape). In other words, strong centrifugation favors the separation of relatively thicker (vertically) and narrower (laterally) flake shapes. Such apparently counterintuitive centrifugation effect, which has also been reported before $^{7,8}$, is probably related to larger frictional drag coefficient of prolate objects (more "1D-like" shaped) in comparison to oblate objects (more "2D-like") 22,23.

We consider now the $\hat{r}_{\text {MinF }} / r_{\mathrm{h}}$ vector, which is actually proportional to the flakiness parameter $f$ ( $f=$ minimum caliper/ height) that can be associated with a two-dimensional shape tendency of a given flake ${ }^{9}$. In other words, the larger the flakiness (or the $\hat{r}_{\text {MinF }} / r_{\mathrm{h}}$ value), the larger the $2 \mathrm{D}$ shape aspect of a flake (see pink arrow on the light orange plane in Fig. $2 \mathrm{~g})^{9}$. Therefore, the $\hat{r}_{\text {MinF }} / r_{\mathrm{h}} \times \hat{r}_{\mathrm{h}} / \mathrm{r}_{\text {Feret }}$ plane (light orange shades in Fig. $2 \mathrm{~g}$ ) directly shows 2D/3D flake shape trends among the four systems: the graphene Parent system has the largest flakiness parameter, or the most "2D-like" character, combined with the smallest voluminosity, or "3D-like" character. In other words, among the four systems investigated here, this is the one, which is mostly comprised by flakes with a "2D-like" shape. And again, heavy centrifugation decreases the flakiness and increases the voluminosity of graphene flakes (17 kg system). At the other end of the flakiness vector, the Talc-US flakes show the smallest "2D-like" shape and the largest "3D-like" shape. To put it another way, there is a lot of room for improvement in this sonication-based process if majoritarily 2D-shaped flakes are to be expected. Finally, the $\hat{r}_{\text {Feret }} / r_{\text {MinF }} \times \hat{r}_{\text {MinF }} / r_{\mathrm{h}}$ plane (dark yellow shades in Fig. $2 \mathrm{~g}$ ) correlates the flakiness of a system with its in-plane asymmetry (or tendency for a dominant in-plane directionality-the "1D-like" or "ribbonlike" shape). Both graphene systems are more "2D-shaped" than the talc systems and, within the graphene systems, the $17 \mathrm{~kg}$ flakes have a larger in-plane asymmetry (or "1D-shape") than the Parent flakes (the centrifugation effect discussed above ${ }^{8,22,23}$ ). On the other hand, both talc systems portray a slightly larger in-plane symmetry (or smaller "1D-shape") than both graphene systems.
The Talc-Sh flakes are probably the most three-dimensionally symmetrical (isotropic) among all four systems studied here.

It is important to stress here a difference between shape and size of a flake when defining its "1D-", "2D-", and "3D-like" character. For example, a conventional (and macroscopic) dinner plate certainly has a large flakiness parameter (or a "2D-like" shape). However, due to its thickness, it hardly can be defined as a true 2D object. Therefore, one should keep in mind that the above discussion is shape-related and not size-related. For example, as said above, graphene Parent flakes portray the largest flakiness, or 2D-like shape. But they are not necessarily the thinnest flakes (or truly 2D flakes-mono- or few-layered). As shown in Fig. 2a, the Parent sample has a large number of thick (large $h$ ) flakes in comparison with the centrifuged $17 \mathrm{~kg}$ sample. A large flakiness (or "2D-like" shape) simply tells that an object has two characteristic dimensions much larger than its third characteristic dimension (as a dinner plate) regardless of their respective size values.

So far, the analysis of the topological vectors presented here relies on the most-probable values, or the peak positions, of the distributions shown in Fig. 2c, d, e. Nevertheless, this topological vector system can also be used to characterize the asymmetry of each distribution curve. The asymmetry of the EMG distribution (or its "shape" parameter $\mathrm{k}$ ) is related to its skewness $\gamma$, or a combination of the $\lambda$ and $\sigma$ values of the distribution, through the definition $\mathrm{k}=(\sigma \lambda)^{-124}$. Using the mathematical relation between standard deviation $s$ and skewness $\gamma$ with $\sigma$ and $\lambda$, it is easy to see that $\mathrm{k}$ is a function of the distribution skewness $\gamma$ only ${ }^{20}$ :

$$
k=(\sigma \lambda)^{-1}=\frac{(\gamma / 2)^{1 / 3}}{1-(\gamma / 2)^{2 / 3}}
$$

which is a monotonically increasing function of $\gamma$ for $\gamma<2$. A Gaussian distribution has $\gamma=0$, whereas an exponential distribution has $\gamma=2^{20}$. The smaller the k value, the closer to Gaussian (or more symmetric) the distribution. Alternatively, the larger the $\mathrm{k}$ value, the more asymmetric (or skewed) the distribution.

Figure 3a shows $k$-shape vectors for all systems. It is evident to see that the Talc-US system has the most asymmetric distributions (larger vector components) - which is in accordance with the plots in Fig. 2. At the other end, the Talc-Sh system has the most symmetrical distributions. It is interesting to observe as well on the graphene systems that centrifugation makes the distribution less symmetric in the lateral dimension directions (larger $k_{\text {Feret }}$ and $\mathrm{k}_{\mathrm{MinF}}$ ), but slightly more symmetrical on the vertical (thicknessrelated) direction $k_{h}$. Yet another interesting shape parameter for the EMG distribution can be defined: its trimness $\boldsymbol{\tau}$, where trimness is defined here as a combination of both asymmetry and variance. The trimness vector components are related to both skewness $\gamma$ and standard deviation s of the EMG distribution and can be calculated via the following definition:

$\tau=\frac{\sigma}{\lambda}=s^{2}\left[(\gamma / 2)^{2 / 3}-(\gamma / 2)^{4 / 3}\right]^{1 / 2}$

Trimness is a monotonically increasing function of both standard deviation $s$ and skewness $\gamma$ (for $\gamma<1.7$ ). In this case, a small trimness value indicates both a symmetric (gaussian-like) distribution with a small width (or small variance)—or a "trimmed" shape. Figure $3 \mathrm{~b}$ shows trimness vectors for all four systems investigated here. It is interesting to observe that the Talc-Sh system shows the best combination of small asymmetry and small variance of them all. The Talc-US system, on the other hand, albeit being the most asymmetrical (see Fig. 3a), has trimness values comparable with the more symmetrical graphene samples. This is due to its smaller variance (or smaller distribution width). Within the graphene systems, centrifugation trims the $r_{h}$-related distribution, but spoils both $r_{\text {Feret }}$ and $r_{\text {MinF }}$-related distributions.

We recall now the brief size/shape difference discussed above. We must remember that any dimensionless aspect ratio, like $r_{h}$, 
a

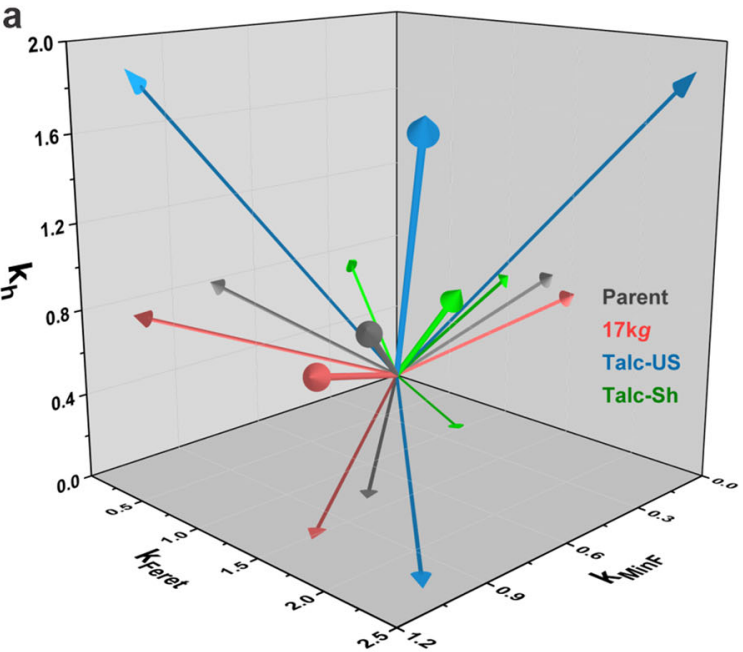

C

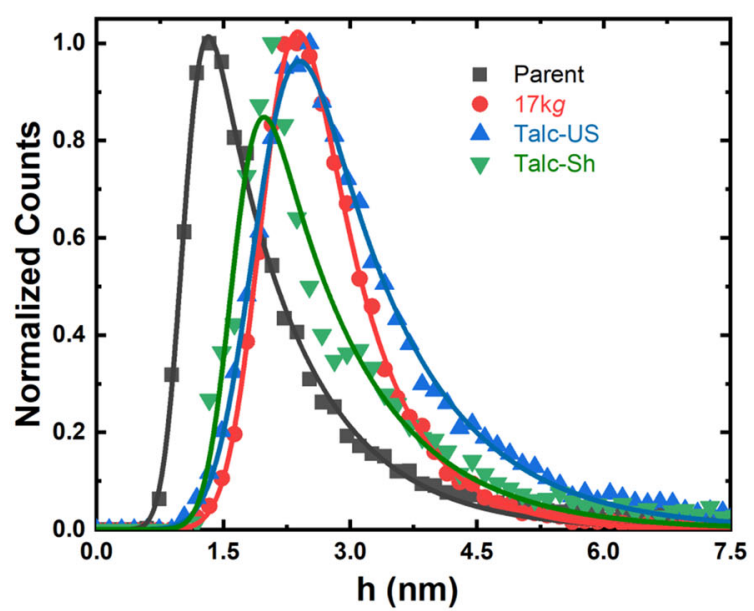

e

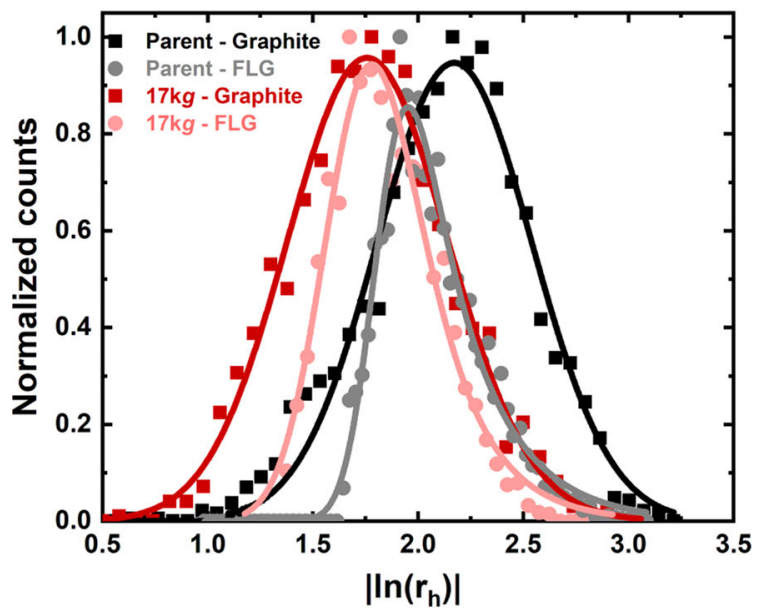

b

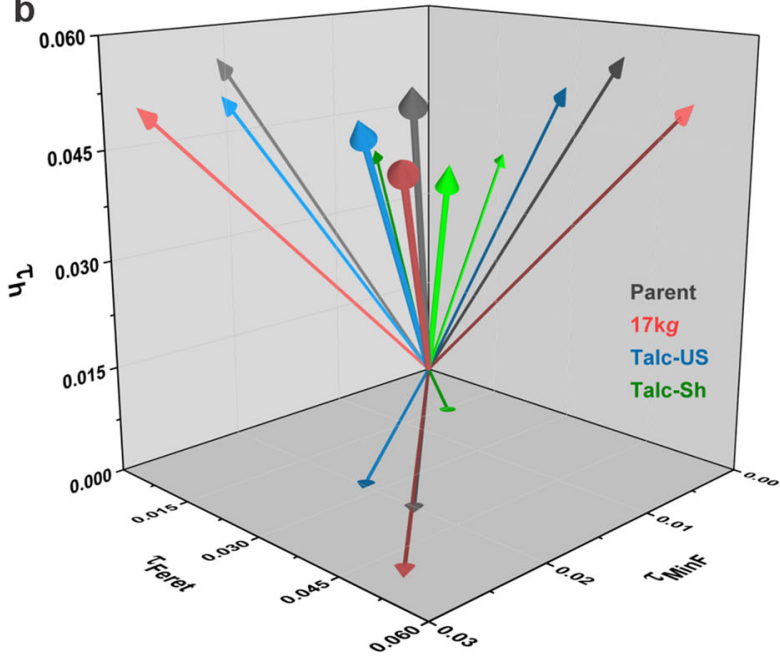

d

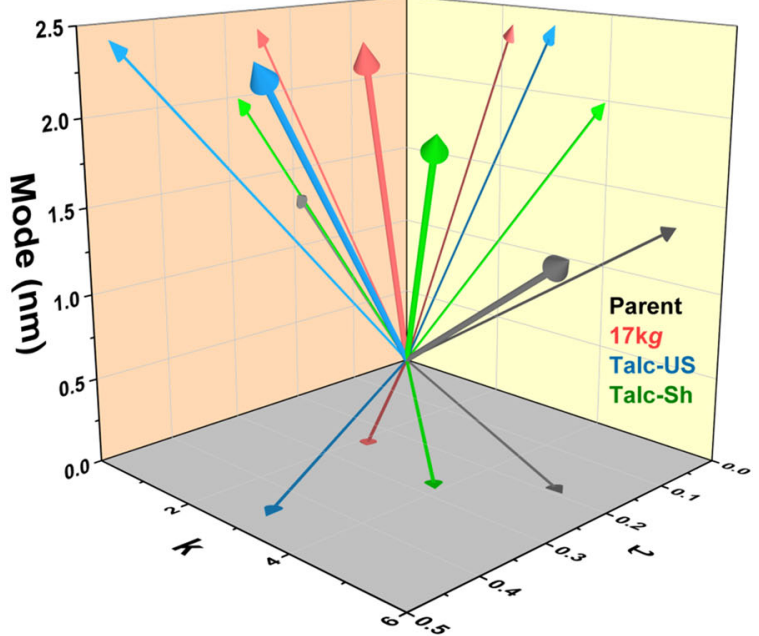

f

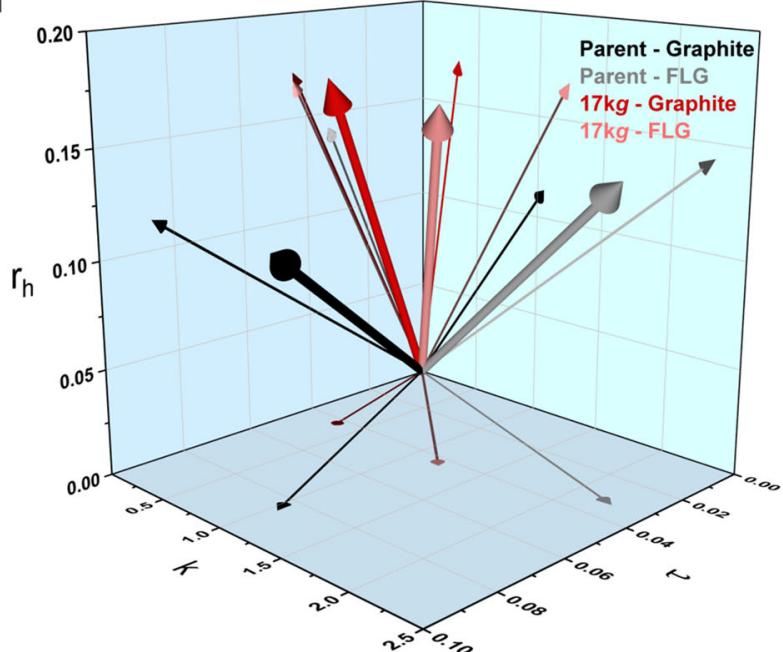

Fig. 3 Distribution shape vectors $k$ and $\tau$ and normalized distributions of flake thickness $h$ and partitioned $r_{h}$, including their respective vectorial representations. a $k$-shape vectors with components $k_{h}, k_{F e r e t}$, and $k_{\text {Minf }}$ for all four systems. $\mathbf{b}$ Trimness vectors with components $\tau_{h}$ $\tau_{\text {Feret, }}$ and $\tau_{\text {Minf }}$ for all four systems. c Normalized histogram of flake thickness $\mathbf{h}$ for each system. $\mathbf{d}$ Vectorial plot of the three characteristic parameters of each distribution: mode, $\mathrm{k}$ and $\tau$. e: Normalized histogram of $\ln \left(\mathbf{r}_{\mathbf{h}}\right)$ for the Parent and $17 \mathrm{~kg}$ samples partitioned into FLG and graphite flakes. In Panel a through d: Parent-black; $17 \mathrm{~kg}$-red; Talc-US—blue and Talc-Sh-green. In panels e and f: Parent-Graphite-black; Parent-FLG—Gray; $17 \mathrm{~kg}$-Graphite—red; $17 \mathrm{~kg}$-FLG—pink. The projections along each plane are also shown in a, b, d, f. 
$r_{\text {Feret }}$ and $r_{\text {MinF, }}$ or any other we may define using ratios of sizeable quantities, are, by definition, shape descriptors and not size descriptors. In other words, using such dimensionless parameters, one can infer a lot of information about the shape of a characteristic flake, but not about its size. Size analysis must be discussed, thus, using pure size quantities separately, such as flake thickness $\mathbf{h}$ or its maximum caliper Feret. Nevertheless, all the methodology developed in this work, including the vectorial representation, is also useful in the description of size parameters. Flake thickness $\mathbf{h}$ was chosen to illustrate such applicability and Figs. $3 c$ and $3 d$ summarize it. Figure $3 c$ shows the normalized histogram distribution of the thickness $\mathbf{h}$ for more than 10,000 flakes of each sample investigated here. A peaked skewed distribution is found for all cases and the EMG distribution efficiently fits them all $\left(R^{2}>0.99\right.$ for Parent, $17 \mathrm{~kg}$ and Talc-US samples and $\mathrm{R}^{2}>0.96$ for Talc-Sh). Therefore, we can also construct the vectorial space representation shown in Fig. $3 \mathrm{~d}$, which digests all the information in the histograms and expedites their comparison. In such representation, the three -vector components of a distribution are: mode (or its most-probable height value), $k$ shape (its symmetry) and trimness $\tau$ (its symmetric spread). The resulting vectors and their respective projection along the defined planes enable a multitude of analyses. For illustration purposes, we will retain our attention to the mode versus $k$ plane for the graphene samples (light yellow shades in Fig. 3d) and to the mode versus trimness plane for the talc samples (light orange shades in Fig. 3d). Beginning with the talc samples, both LPE protocols (ultrasound or shear) yield similar results in terms of flake thickness, with the most-probable flake being a talc bilayer ${ }^{25}$. However, the Talc-Sh distribution is more trimmed than the Talc-US (smaller $\tau$ ) and, therefore, has a significant smaller number of thick flakes (smaller distribution tails). In other words, for industrial application purposes, the Talc-Sh sample may be the best option because, flake thickeness-wise, it is a more homogeneous sample. Considering now the graphene samples and because a pure size parameter is being analyzed (flake thickness $\mathbf{h}$ ), one can safely say that the average flake thickness actually increases after centrifugation, instead of a decrease that is normally reported in the literature ${ }^{7}$. Such apparent discrepancy is explained by different centrifugation protocols: while several works employ cascade centrifugation, which readily yields a decreasing thickness with centrifugation ${ }^{7}$, a single step centrifugation with high acceleration is used here and only the supernatant part is analyzed. As a consequence, a strong hydrodynamical drag caused by large graphite flakes being accelerated downward also pulls tiny graphene flakes downward upon strong centrifugation. Thus, not only large graphite flakes are filtered out, but also the small graphene ones (see also Fig. $2 a)^{8}$. As a result, the Parent graphene sample has the thinnest flakes of this sample set, with a large number of truly 2-dimensional flakes (less than 5 graphene layers, also called few-layer graphene - FLG). However, it shows the most asymmetric distribution, with a long tail of thick graphite flakes. Even though centrifugation significantly removes FLG flakes, it also makes their thickness distribution more symmetrical by removing thick graphite flakes as well. To further compare FLG and graphite flakes in both systems and taking advantage of their large number of measured flakes, both systems were repartitioned into three categories according to the number of graphene layers in a flake: FLG (between 1 and 5 layers), multilayer graphene (between 6 and 10 layers) and graphite (above 10 layers). Each partition comprises several thousand flakes and, for illustration purposes, the $r_{h}$ shape parameter was analyzed for both FLG and graphite partitions in both Parent and $17 \mathrm{~kg}$ systems (Fig. 3e, f). The fact that the Parent sample has the most 2D flakes in number $(\sim 1 / 3$ of flakes are FLG in comparison with $\sim 1 / 4$ of FLG flakes in the $17 \mathrm{~kg}$ sample) does not mean that it also has the largest amount of 2D flakes in mass. As discussed by Fernandes et al., a small fraction of thick flakes ( 15\% for the Parent sample), can actually represent the vast majority of the mass of a given sample ${ }^{13}$. In other words, the $17 \mathrm{~kg}$ centrifuged graphene sample (with only $\sim 8 \%$ of graphite flakes) may actually be a better fit for industrial applications due to the absence of large and thick graphite flakes, which are produced during the initial phases of LPE and may dominate the sample mass ${ }^{13}$. Additionally, Fig. 3e, $f$ bring interesting observations: (i) as expected, the vertical shape morphology $r_{h}$ is smaller (thinner flakes) for FLG flakes than for graphite flakes in the Parent sample. However, for the centrifuged sample, both FLG and graphite flakes portray similar most-probable morphology (peak positions in Fig. 3e). This is another unexpected shape selection effect from centrifugation, as those discussed in Fig. 2g. (ii) Regarding the symmetry parameter $k$, the graphite shape distribution is much more symmetrical (smaller $k$ values in Fig. $3 f$ ) than the FLG distribution for both Parent and $17 \mathrm{~kg}$ systems. Since FLG flakes are a result of many more breaking-and-tear processes than graphite flakes, one should expect, indeed, a higher asymmetry of shape distribution in FLG flakes in comparison with graphite flakes within a given system. (iii) Regarding the trimness $\tau$ of the distributions, in the Parent system, FLG distribution is more trimmed (normally a combination of larger symmetry and smaller dispersion) than the graphite distribution. But since, as discussed above, FLG flake distribution is actually more asymmetrical than the graphite distribution (the $\mathrm{k}$ parameter), the smaller trimness in FLG is a result of a much smaller dispersion (see Fig. 3e), which compensates for its larger asymmetry. For the $17 \mathrm{~kg}$ system, both FLG and graphite $\ln \left(r_{h}\right)$ distributions show similar trimness, which is a result of the larger symmetry of the graphite distribution and the smaller dispersion of the FLG distribution. This is yet another interesting shape selection effect of centrifugation.

Finally, we discuss on the possible origin of the exponentially modified Gaussian distributions observed in the analyses of size and shape parameters of flakes produced by LPE. It has been suggested that particle size $\beta$ (where $\beta$ may denote thickness, length or width), produced via several comminution processes such as milling, grinding or vibration, generally follows a lognormal distribution $^{26}$. As a consequence, the logarithm of any size parameter $\ln (\beta)$ should follow a normal distribution. The physical origin of the lognormal distribution is the random nature of the breaking events leading to the formation/detachment of a small "offspring" particle with characteristic size $\beta_{1}$ from a large "parent" body with characteristic size $\beta_{0}\left(\beta_{1}<\beta_{0}\right)^{26}$. Suppose now that an additional secondary process is possible: the breaking of the first generation "offspring" particles into smaller second-generation "offspring" particles. Obviously, the characteristic size $\beta_{1}$ of the first generation "offspring" particle limits the respective size $\beta_{2}$ of the second-generation "offspring" ( $\beta_{2}$ cannot be larger than $\left.\beta_{1}\right)$. The number $\mathrm{N}_{2}\left(\beta_{2}\right)$ of second-generation particles with characteristic size $\beta_{2}$ in this secondary process is, thus, proportional to the number $N_{1}\left(\beta_{1}\right)$ of first generation "offspring" particles with the constraint $\beta_{2}<\beta_{1}$. Clearly, this process can continue in tertiary, quaternary and so forth processes $(i=2,3,4 \ldots)$. In all cases, $N_{i+1}\left(\beta_{i+1}\right)$ $\propto N_{i}\left(\beta_{i}\right)$ with the constraint $\beta_{i+1}<\beta_{\mathrm{i}}$. Therefore, such chain of processes should yield a given number $N$ of particles with an exponential-type law with decreasing size $\beta$. In other words, sequential events should create a large number of small objects (small $\beta$ ) with an exponential decrease in the number of particles $\mathrm{N}$ as $\beta$ gets larger. The overall process of particle creation is, thus, the sum of both primary and chain mechanisms. In this way, the distribution of the logarithm of size parameters of the generated particles should be the convolution of a normal distribution process with an exponential-type process, which is the definition of an exponentially modified Gaussian distribution.

In summary, the topological vector methodology developed here trails a few steps on the route towards thorough and meaningful characterization of 2D material flakes. Initially, a new set of shaperelated characteristic flake parameters is proposed. Its association with a well-chosen distribution function enables a good evaluation 
of each flake shape distribution, also describing quantitatively its asymmetry and dispersal. Then, a new graphical description system is presented-the topological vectors-, which facilitates the simultaneous visualization and comparison of several characterization parameters. All these new methodological features were illustrated with a brief analysis of two typical 2D materials: graphene and talc, which enabled interesting insights into their flake morphology. The methodology introduced here forms a complete visual toolset to efficiently characterize, or fingerprint, industrialscale amounts of different LPE-produced 2D materials. Therefore, it enables an efficient comparison between 2D systems, creating both a trustful positive feedback into production protocols and a universal representation candidate to be easily implemented across the entire 2D-materials industry.

\section{METHODS}

\section{Sample preparation for AFM measurements}

A bare $\mathrm{SiO}_{2} / \mathrm{Si}$ substrate is immersed for 15 minutes into an aqueous solution of (3-aminopropyl)triethoxysilane-APTES). Subsequently, the substrate is repeatedly cleaned with DI water and then dried with an $\mathrm{N}_{2}$ blow. Nanoflake samples are prepared by drip-coating the APTESfunctionalized substrate with the appropriate nanoflakes' solution (graphene or talc) for $20 \mathrm{~s}$. Subsequently, the drop is discarded, and the sample is repeatedly cleaned with DI water before being finally dried with an $\mathrm{N}_{2}$ blow to remove any residual surfactants ${ }^{13}$.

\section{AFM measurements and analysis}

The AFM characterization was carried out on Park NX10 and Park XE-70 microscopes using the silicon cantilever HQ:NSC35/AIBS or HQ:NSC36/AIBS (MikroMasch), in intermittent contact mode with a free amplitude of $30 \mathrm{~nm}$ and a setpoint of $15 \mathrm{~nm}$. The region to be measured was chosen randomly and image lateral sizes of $5 \mu \mathrm{m}$ with $500 \times 500$ pixels, $10 \mu \mathrm{m}$ with $1000 \times$ 1000 pixels or $15 \mu \mathrm{m}$ with $1500 \times 1500$ pixels at $0.5 \mathrm{~Hz}$ scan rate were used in this work. The generated AFM images are plane-corrected using the Gwyddion software. Individual flake analysis for the AFM images was performed using ImageJ particle analysis toolbox, where flake dimensions (height, maximum and minimum calipers and volume) are acquired ${ }^{13}$.

\section{Production of LPE graphene samples}

The graphene Parent dispersion was obtained through high-shear exfoliation of graphite dispersed in water with a nonionic surfactant for stabilization. The experimental arrangement consists of a high-shear mixing connected to a $100 \mathrm{~L}$ tank. The material was continuously processed overnight at room temperature. The stratification process of the Parent dispersion was performed through direct centrifugation using a Hettich Rotanta $460 \mathrm{R}$ centrifuge. For the $17 \mathrm{~kg}$ acceleration sample, a fresh aliquot of the Parent solution was placed in a $50 \mathrm{~mL}$ Falcon tube and centrifuged for 1 hour. Afterwards, the supernatant was separated from the sediment by pipetting $15 \mathrm{~mL}$ of the dispersion from the top of the dispersion.

\section{Production of LPE talc samples}

Talc was obtained through a private donation of a steatite crystal from Minas Gerais state, Brazil. The rock was milled to a fine powder and X-ray diffraction (XRD) was performed to characterize the sample's composition ${ }^{25}$. Part of this talc powder was used for production of the Talc-Sh sample and part for the Talc-US sample. The Talc-Sh sample was produced via a shear process similar to the graphene Parent sample. For the sonication-based talc sample, the following protocol was employed: Deionized water (resistivity $18.2 \mathrm{M} \Omega . \mathrm{cm}$ ) from a MiliQ system was used for solution preparation. A commercial surfactant, Triton ${ }^{T M}-\mathrm{X} 100$, was purchased from Sigma-Aldrich and used as received. Before submitting the material to the liquid exfoliation process, a purification step was performed to remove any contamination ${ }^{27}$. Talc powder was sonicated for $10 \mathrm{~min}$ in chloroform and then the solution was left to decant. Supernatant was discarded and the process was repeated for acetone and water. Finally, the powder was collected and dried for $12 \mathrm{~h}$ in an oven at $60^{\circ} \mathrm{C}$. Purified talcum powder was placed in an aqueous Triton ${ }^{\mathrm{T} M}-\mathrm{X} 100$ solution ( $6 \mathrm{mg} / \mathrm{ml} \mathrm{Talcum/liquid}$ and $1 \mathrm{mg} / \mathrm{ml} \mathrm{Triton}^{\mathrm{TM}}-\mathrm{X} 100$ to DI water). Glass vials containing the solutions were placed in an ultrasonic bath (Elma, $\mathrm{S} 1 \mathrm{OH}$ ) for 15 hours. The bath water temperature was monitored and controlled with ice additions to keep it below $40^{\circ} \mathrm{C}$. The resulting solutions were centrifuged at
$1 \mathrm{~kg}$ for $1 \mathrm{~h}$ (Sorval Legend Mach 1.6) to remove non-exfoliated materia ${ }^{28}$. All analyses were performed with the collected supernatant.

\section{Relation between EMG parameters and distribution mean, standard deviation and skewness}

The three parameters $\mu, \sigma$, and $\lambda$ of the EMG function can be directly calculated from the extracted values of the distribution mean $m$, standard deviation $s$ and skewness $\gamma$ via:

$$
\begin{aligned}
& \mu=m-s\left(\frac{\gamma}{2}\right)^{1 / 3} \\
& \sigma=s \sqrt{\left[1-\left(\frac{\gamma}{2}\right)^{2 / 3}\right]}
\end{aligned}
$$

and

$\lambda^{-1}=s\left(\frac{\gamma}{2}\right)^{1 / 3}$

The position $x_{M}$ of the Mode (peak top position-or the most-probable value of the distribution) can be calculated via:

$x_{M}=\mu+\lambda \sigma^{2}-\sqrt{2} \sigma \xi$

Where $\xi$ is the solution (root) of the equation

$$
\operatorname{erfcx}(\xi)=\frac{1}{\lambda \sigma} \sqrt{\frac{2}{\pi}}
$$

And $\operatorname{erfcx}(\mathrm{x})$ is the scaled complementary error function.

\section{DATA AVAILABILITY}

The datasets generated during and/or analysed during the current study are available from the corresponding author on reasonable request.

Received: 22 January 2021; Accepted: 16 April 2021; Published online: 14 May 2021

\section{REFERENCES}

1. Ferrari, A. C. et al. Science and technology roadmap for graphene, related two dimensional crystals, and hybrid systems. Nanoscale 7, 4598-4810 (2015).

2. Yi, M. \& Shen, Z. A review on mechanical exfoliation for the scalable production of graphene. J. Mater. Chem. A 3, 11700-11715 (2015).

3. Nicolosi, V., Chhowalla, M., Kanatzidis, M. G., Strano, M. S. \& Coleman, J. N. Liquid exfoliation of layered materials. Science 340, 1226419 (2013).

4. Backes, C. et al. Production and processing of graphene and related materials. $2 D$ Mater. 7, 022001 (2020).

5. Ciesielski, A. \& Samori, P. Graphene via sonication assisted liquid-phase exfoliation. Chem. Soc. Rev. 43, 381-398 (2014).

6. Kouroupis-Agalou, K. et al. Fragmentation and exfoliation of 2-dimensional materials: a statistical approach. Nanoscale 6, 5926-5933 (2014).

7. Backes, C. et al. Equipartition of energy defines the size-thickness relationship in liquid-exfoliated nanosheets. ACS Nano 13, 7050-7061 (2019).

8. Chacham, H. et al. Controlling the morphology of nanoflakes obtained by liquidphase exfoliation: implications for the mass production of 2D materials. ACS Appl. Nano Mater. 3, 12095-12105 (2020).

9. Merkus, H. G. Particle Size Measurements: Fundamentals, Practice, Quality (Springer, 2009)

10. Erasmus, J. J., Connolly, J. E., McAdams, H. P. \& Roggli, V. L. Solitary pulmonary nodules: Part I. Morphologic evaluation for differentiation of benign and malignant lesions. Radiographics 20, 43-58 (2000).

11. Mogilner, J. G. et al. Computer-assisted image analysis can aid the prognostication of advanced-stage neuroblastomas. J. Cancer Res. Clin. Oncol. 126, 285-290 (2000).

12. Yap, F. Y. et al. Quantitative morphometric analysis of hepatocellular carcinoma: development of a programmed algorithm and preliminary application. Diagn. Interv. Radiol. 19, 97-105 (2013).

13. Fernandes, T. D. F. et al. A semi-automated general statistical treatment of graphene systems. 2D Mater. 7, 025045-025047 (2020).

14. Wackerly, D., Mendenhall, W. \& Scheaffer, R. Mathematical Statistics with Applications. 7th edn. (Thomson Brooks/Cole, 2007).

15. McWilliam, I. G. \& Bolton, H. C. Instrumental peak distortion. I. Relaxation time effects. Anal. Chem. 41, 1755-1762 (1969). 
16. Grushka, E. Characterization of exponentially modified Gaussian peaks in chromatography. Anal. Chem. 44, 1733-1738 (1972).

17. Golubev, A. Exponentially modified Gaussian (EMG) relevance to distributions related to cell proliferation and differentiation. J. Theor. Biol. 262, 257-266 (2010).

18. Gunzert-Marx, K. et al. Secondary beam fragments produced by $200 \mathrm{MeVu}-112 \mathrm{C}$ ions in water and their dose contributions in carbon ion radiotherapy. N. J. Phys. 10, 1-21 (2008)

19. Cricenti, A. L. \& Branch, P. A. The Ex-Gaussian distribution as a model of firstperson shooter game traffic. Multimed. Syst. 19, 221-229 (2013).

20. Olivier, J. \& Norberg, M. M. Positively skewed data: revisiting the box-cox power transformation. Int. J. Psych. Res. 3, 68-75 (2010).

21. Li, Z. et al. Mechanisms of liquid-phase exfoliation for the production of graphene. ACS Nano 14, 10976-10985 (2020).

22. Tillett, J. P. K. Axial and transverse Stokes flow past slender axisymmetric bodies. J. Fluid Mech. 44, 401-417 (1970).

23. Leung, W. W.-F. Centrifugal separations in Biotechnology (Elsevier, 2020).

24. Foley, J. P. \& Dorsey, J. G. A review of the exponentially modified Gaussian (EMG) function: evaluation and subsequent calculation of universal data. J. Chromatogr. Sci. 22, 40-46 (1984).

25. Alencar, A. B., Barboza, A. P. M., Archanjo, B. S., Chacham, H. \& Neves, B. R. A. Experimental and theoretical investigations of monolayer and few-layer talc. $2 D$ Mater. 2, 015004 (2015)

26. Neikov, O. D., Naboychenko, S. S. \& Yefimov, N. A. Handbook of Non-Ferrous Metal Powders-Technologies and Applications (Elsevier, 2019).

27. Harvey, A. et al. Exploring the versatility of liquid phase exfoliation: producing 2D nanosheets from talcum powder, cat litter and beach sand. 2D Mater. 4, 025054 (2017).

28. Backes, $C$. et al. Production of highly monolayer enriched dispersions of liquidexfoliated nanosheets by liquid cascade centrifugation. ACS Nano 10, 1589-1601 (2016).

\section{ACKNOWLEDGEMENTS}

We thank the MGgrafeno initiative for producing the graphene samples. We also acknowledge financial support from Fapemig, CNPq, INCT-NanoCarbono, and PRPPGIFMG. The MGgrafeno initiative is funded by Codemge.

\section{AUTHOR CONTRIBUTIONS}

J.C.C.S. and M.C.P. carried out the AFM imaging; M.C.P., H.L.O.M., S.M.S., and E.S.P. prepared talc samples; L.G.C. provided graphene samples and analyzed some results; B.R.A.N. proposed the project, analyzed the results, and wrote the paper with inputs from all authors.

\section{COMPETING INTERESTS}

The authors declare no competing interests.

\section{ADDITIONAL INFORMATION}

Correspondence and requests for materials should be addressed to B.R.A.N.

Reprints and permission information is available at http://www.nature.com/ reprints

Publisher's note Springer Nature remains neutral with regard to jurisdictional claims in published maps and institutional affiliations.

(i) Open Access This article is licensed under a Creative Commons c. Attribution 4.0 International License, which permits use, sharing, adaptation, distribution and reproduction in any medium or format, as long as you give appropriate credit to the original author(s) and the source, provide a link to the Creative Commons license, and indicate if changes were made. The images or other third party material in this article are included in the article's Creative Commons license, unless indicated otherwise in a credit line to the material. If material is not included in the article's Creative Commons license and your intended use is not permitted by statutory regulation or exceeds the permitted use, you will need to obtain permission directly from the copyright holder. To view a copy of this license, visit http://creativecommons. org/licenses/by/4.0/.

(c) The Author(s) 2021 